\title{
Measuring Nonlinear Momentum Compaction in RHIC*
}

\author{
M. Blaskiewicz ${ }^{\dagger}$, J.M. Brennan, P. Cameron, A. Drees, \\ J. Kewish, T. Roser, K. Smith, C. Tang \\ BNL, Upton NY 11973, USA
}

\section{Abstract}

The chromatic nonlinearity parameter, $\alpha_{1}$, has a strong impact on longitudinal dynamics in the vicinity of transition $[1,2,3]$. Measurements of the synchrotron frequency as a function of radius are used to constrain the value of $\alpha_{1}$.

\section{PRELIMINARIES}

The lattice parameters $\alpha_{0}$ and $\alpha_{1}$ relate the change in closed orbit path length $C$ with the reference value for the center of the beam pipe $C_{0}$ and the fractional momentum difference between the closed orbit momentum $p$ and the reference value for the center of the beam pipe $p_{0}$ via $[1,2$, 3]:

$$
\frac{C}{C_{0}}=1+\alpha_{0} \delta\left(1+\alpha_{1} \delta\right)+O\left(\delta^{3}\right)
$$

where $\delta=\left(p-p_{0}\right) / p_{0}$ is the fractional momentum difference of the closed orbit and reference orbit momenta. To get the revolution frequency one needs the change in velocity $(\beta c)$ between the closed and reference orbits.

Define $u=p / m c$ then :

$$
\begin{aligned}
\frac{1}{\beta} & =\left(1+u^{-2}\right)^{1 / 2} \\
\frac{d}{d u} \frac{1}{\beta} & =-u^{-3}\left(1+u^{-2}\right)^{-1 / 2} \\
\frac{d^{2}}{d u^{2}} \frac{1}{\beta} & =3 u^{-4}\left(1+u^{-2}\right)^{-1 / 2} \\
& -u^{-6}\left(1+u^{-2}\right)^{-3 / 2}
\end{aligned}
$$

Since $\delta=\left(u-u_{0}\right) / u_{0}$

$$
\frac{1}{\beta}=\frac{1}{\beta_{0}}\left(1-\frac{\delta}{\gamma_{0}^{2}}+\frac{\delta^{2}}{2}\left[\frac{3}{\gamma_{0}^{2}}-\frac{1}{\gamma_{0}^{4}}\right]\right)+O\left(\delta^{3}\right)
$$

The revolution period is $T=C / \beta c$ so

$$
\begin{aligned}
\frac{T}{T_{0}} & =1+\left(\alpha_{0}-\frac{1}{\gamma_{0}^{2}}\right) \delta \\
& +\delta^{2}\left(\alpha_{0} \alpha_{1}-\frac{\alpha_{0}}{\gamma_{0}^{2}}+\frac{3}{2 \gamma_{0}^{2}}-\frac{1}{2 \gamma_{0}^{4}}\right)+O\left(\delta^{3}\right)(6)
\end{aligned}
$$

The data are synchrotron frequency versus radius. These were obtained with the $2 \mathrm{GHz}$ Schottky cavity and the values at the peaks in the synchrotron spectrum correspond to

\footnotetext{
* Work performed under the auspices of the United States Department of Energy

†blaskiewicz@bnl.gov
}

small amplitude synchrotron oscillations. Therefore, a linear expansion of the equations of motion about the stable fixed point will suffice. Define

$$
\hat{\delta}=\frac{p-p_{s}}{p_{0}}=\frac{p-p_{0}}{p_{0}}-\frac{p_{s}-p_{0}}{p_{0}}=\delta-\delta_{s}
$$

where $p_{s}$ is the synchronous momentum, and let $\tau=T-$ $T_{s}$ be the difference in revolution period between a particle and the synchronous particle. The experiment was done at constant magnetic field below transition so:

$$
\begin{aligned}
\frac{d \hat{\delta}}{d n} & =\frac{q V}{\gamma_{0} \beta_{0} \beta_{s} m c^{2}}\left(\omega_{r f} \tau\right) \\
\frac{d \tau}{d n} & =T_{0} \hat{\delta}\left(\frac{d}{d \delta} \frac{T}{T_{0}}\right)_{\delta=\delta_{s}}
\end{aligned}
$$

where $n$ is the turn number. The synchrotron frequency is given by

$$
f_{s}^{2}=\frac{q V}{\gamma_{0} \beta_{0} m c^{2}} \frac{\omega_{r f}^{3}}{4 \pi^{2} h^{2}} \frac{T_{0}}{\beta_{s}}\left(\frac{d}{d \delta} \frac{T}{T_{0}}\right)_{\delta=\delta_{s}},
$$

where $h=360$ is the harmonic number. In equation (10) only $\omega_{r f f}, \beta_{s}$ and the derivative term vary with radial steering and they are tightly related since

$$
\frac{\omega_{r f}}{\omega_{r f, 0}}=\frac{T_{0}}{T_{s}}
$$

Taking the logarithm of equation (10), differentiating with respect to $\delta_{s}$, and evaluating at $\delta_{s}=0$ yields

$$
\begin{aligned}
\frac{2}{f_{s}} \frac{d f_{s}}{d \delta_{s}} & =2 \frac{\alpha_{1}+\frac{3}{2 \gamma_{0}^{2} \alpha_{0}}+O\left(\alpha_{0}\right)}{1-\frac{1}{\gamma_{0}^{2} \alpha_{0}}} \\
& -3\left(\alpha_{0}-1 / \gamma_{0}^{2}\right)-\frac{1}{\gamma_{0}^{2}} .
\end{aligned}
$$

In equation (12) the $O\left(\alpha_{0}\right)$ appearing in the numerator of the first term on the right are found in equation (6) and are neglected since they produce a very small correction. Also the second and third terms on the right of equation (12) are very small near transition and will be neglected.

\section{APPLICATION}

The data for the yellow ring and a least squares fit are shown in Figure 1. The first measurement was at $x=0$ 


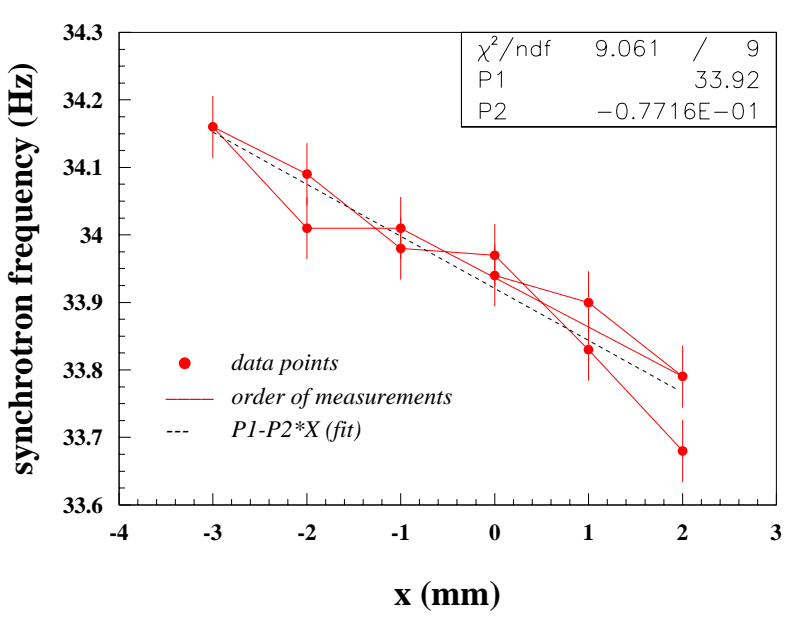

Figure 1: Measured synchrotron frequency versus radial steering setpoint.

and the second at $x=1 \mathrm{~mm}$. Notice that the chronological order of the measurements went to large then small then large radius. There are no systematic drifts. An ideal $\chi^{2}$ requires $1 \sigma$ errors of $0.046 \mathrm{~Hz}$. The resolution bandwidth of the spectrum analyzer was $1 \mathrm{~Hz}$ and measurements were made using 11 synchrotron lines $\left(10 f_{s}\right)$. The expected error is $\sim \sqrt{2} /(10 \sqrt{12}) \mathrm{Hz}=0.04 \mathrm{~Hz}$, where the $\sqrt{12}$ comes from a boxcar distribution. The factor of 10 comes from a span of $10 f_{s}$ and the additional $\sqrt{2}$ comes from the two independent measurements of synchrotron frequency at the edges of the 11 line span.

The reference value for the energy was $\gamma_{0}=20$ and both the horizontal and vertical chromaticities were $\lesssim 1$. Assume a bare value of $\gamma_{t} \equiv 1 / \sqrt{\alpha_{0}}=22.76$. Assuming the frequency steering is accurate at this value of gamma

$$
\frac{d f_{s}}{d \delta}=R_{0} \alpha_{0} \frac{d f_{s}}{d x}
$$

where $R_{0}=610.2 \mathrm{~m}$ is the reference radius and $x$ is the horizontal position. From the fit to the data

$$
\frac{1}{f_{s}} \frac{d f_{s}}{d \delta_{s}}=-2.68 \pm 0.23
$$

Now since

$$
1-\frac{\gamma_{t}^{2}}{\gamma_{0}^{2}}=-0.295, \quad \frac{3 \gamma_{t}^{2}}{2 \gamma_{0}^{2}}=1.94,
$$

one obtains the final result

$$
\alpha_{1}=-1.15 \pm 0.10 .
$$

The MAD[4] model of the RHIC ring predicts

$$
\alpha_{1}=-1.0 \text { for } Q_{y}^{\prime}=Q_{x}^{\prime}=0
$$

and

$$
\alpha_{1}=-1.2 \text { for } Q_{y}^{\prime}=Q_{x}^{\prime}=2 .
$$

Given natural chromaticities $Q_{x}^{\prime} \sim Q_{y}^{\prime} \sim-30$, the results are in fair agreement with the model.

\section{REFERENCES}

[1] K. Johnsen, Proc. CERN Symp. High Energy Acc. and Pion Physics, Vol. 1, p.106, (1956)

[2] J. Wei, PhD Thesis, SUNY at Stony Brook, (1990, rev. 1994).

[3] Handbook of Physics and Accelerator Engineering, Eds. Chao \& Tigner, World Scientific, (1998), p 92.

[4] H. Grote and F.C. Iselin, CERN/SL/90-13, (1995). 